\title{
Street crossing pedestrian detection based on edge curves motion
}

\author{
Abdenbi Mazoul \\ LabSIV, Department of Computer \\ Science, \\ Faculty of Science, University of Ibn \\ Zohr, 80000 Agadir, \\ Morocco.
}

\author{
Khalid Zebbara \\ LabSIV, Department of Computer \\ Science, \\ Faculty of Science, University of Ibn \\ Zohr, 80000 Agadir, \\ Morocco.
}

\author{
Mohamed El Ansari \\ LabSIV, Department of Computer \\ Science, Faculty of Science, \\ University of Ibn Zohr, 80000 \\ Agadir,Morocco \\ LITIS INSA de Rouen, BP 8, 76801 \\ Saint-Etienne-du-Rouvray Cedex,
}

France.

\begin{abstract}
This paper presents a real-time method for detecting pedestrians using vertical motion form two consecutives frames. We used association approach to match edge curves between consecutive images. Significant motions can be found using horizontal-vertical projection histogram. Then the pedestrian detection process is achieved in two steps. The first one searches the region of interest by using the intersection of vertical and horizontal projection of significant motion. The second step applies the Adaboost classifier on the region of interest provided by the first step. The proposed approach has been tested on different city traffic image sequences acquired by a camera mounted in a moving car. The results demonstrate the effectiveness of the proposed method.
\end{abstract}

\section{Keywords}

Pedestrian detection, Image motion analysis, correspondence Edge curves. Adaboost classifier.

\section{INTRODUCTION}

Pedestrian protection is a key technology for many future applications of intelligent vehicles. Large variations in pedestrian appearance (e.g. clothing, pose) and environmental conditions (e.g. lighting, background) make this problem particularly challenging. The goal of our approach is to develop a system with enough speed and accuracy to be useful for detecting pedestrians in real city traffic. The key to achieve this is to detect horizontal motion between corresponding edge curves from two consecutive images. The association approach [14][13] is used to find this correspondence between edge curves. M. El Ansari et al. [12] proposed a novel approach for stereo matching using spatiotemporal matching of edge curves of the consecutive frames. Many other interesting approaches for pedestrian detection have been proposed [8][15].

Adaboost classifier [21][17] is a last step in this process to separate pedestrian from non-pedestrian for each $R O I$ (region of interest). The interesting part of this work is the utilization of Adaboost only for small image windows. An image window is simply a $R O I$ detected in first step using horizontal motion of edge curves. This strategy is very useful to reduce the computational time and to optimize the classification process. Zebbara et al. [24][25] used the same approach for on road vehicle detection.
The set of edge curves that will be used for motion detection are extracted from two consecutive images using Canny edge detector algorithm [9]. Only significant vertical edge curves can be selected. Then the temporal correspondence between curves of consecutive images is computed based on the socalled association [14][13]. False positive are significantly reduced by using Winner-takes-all (WTA) on each corresponding edge curves. The motion velocity can be easily computed by comparing positions of each corresponding edge curves. In this work we propose to use horizontal-vertical motion projection histogram to present significant motions from two consecutive images. Many ROIs (region of interest) can be found from the histogram. Finally the Adaboost classifier has been used to validate pedestrian from nonpedestrian.

The rest of the paper is organized as follows: After reviewing the related work in section 2, section 3 overviews temporal matching of edge curves using association method. The proposed pedestrian detection method is detailed in section 4 . Experimental results are presented in section 5 . We conclude the paper in section 6 .

\section{RELATED WORK}

Pedestrian detection is an essential part of the Intelligent Vehicles Systems. In recent years several techniques have been proposed to obtain accurate detection of pedestrian in traffic environment [2][6][7][16][18][20][22]. Some methods use neural networks [23] or SVM [10][5], others techniques are based on the detection of specific pattern, texture or motion clues [19][11]. Stereo vision based approaches are also used in this topic [4]. A number of sensors embedded in Intelligent Vehicles to perform the objects detection task. These sensors can be classified into passive and active sensors. We propose to use passive sensors in our vehicle detection approach. The data that we are going to process to achieve pedestrian detection are images taken from a camera embedded in a moving car.

This paper describes a modified association-based approach [14] [13] for pedestrian detection. At each time, the decision of the presence of motion in the road scene is made based on the current frame and its preceding one. This method exploits the displacement of edges in the frames. At each edge point in one frame we look for its associate one in the preceding frame. The moving object can be detected on the basis of the analysis of association results. Adaboost classifier is used to verify if an obstacle is a pedestrian. 


\section{TEMPORAL MATCHING OF EDGE CURVES OF CONSECUTIVE IMAGES}

This section describes the method used to match the edge curves of consecutive images. The matching algorithm is achieved in two stages. The first stage finds the possible candidate matches based on the association constraint and the association between edge points of consecutive images. The second stage disambiguates the multiple candidate matches and keeps only the ones that minimize a cost function.

\subsection{Detecting Edge Curves}

In this subsection we used Canny edge detector algorithm [9] to find our edges points, it is regarded as one of the best edge detectors currently in use. It provides continuous edge curves, which are vital to the proposed matching method. We keep only the non-horizontal curves and curves that their lengths are bigger than a threshold, in this paper we fixed a threshold of 5 , that means all curves small that 5 are eliminated.

\subsection{Association Constraint}

In order to lower the combinatorial complexity of the matching problem, the possible homologous edge curves must meet two constraints: the uniqueness constraint and association continuity constraint.

\subsubsection{Uniqueness constraint}

Each feature can at most be associated to one feature in the previous image. That means, one feature in the current image should be not associated to more than one in the precedent image.

\subsubsection{Association continuity constraint}

We consider that the physical surfaces are locally continuous, and this continuity is appeared on both current and next frame. Consequently, a connected edge points in the current image must associate to the connected ones in the previous image. In this work, this constraint is satisfied by using the Winner-takes-all (WTA) algorithm on each edge curves. In other words, the associate points should be belonging to the same object contour which they share the greatest associated points.

\subsection{Edge Curves Matching}

\subsubsection{Association between edge points of consecutive images}

The main idea of the proposed approach is to detect the motion between consecutive images. The authors have proposed the so-called association to achieve this task [14][13]. This subsection describes the method used to find the association between edge points of consecutive frames (i.e., association between edge points of the images $\mathrm{I}_{\mathrm{k}-1}$ and $\mathrm{I}_{\mathrm{k}}$. Let us consider two edge points $\mathrm{P}_{\mathrm{k}-1}$ and $\mathrm{Q}_{\mathrm{k}-1}$ belonging to a curve $C_{k-1}$ in the image $I_{k-1}$ and their corresponding ones $P_{k}$ and $Q_{k}$ belonging to a curve $C_{k}$ in the image $I_{k}$ (see Fig. 1). The associate point to point $\mathrm{P}_{\mathrm{k}-1}$ is defined as the point belonging to the curve $C_{k}$ whith the same y-coordinate as $P_{k-1}$. Two associate points are two edge points belonging to two corresponding curves of two consecutive images of the same sequence and having the same y-coordinate. From Fig. 1, we remark that point $\mathrm{Q}_{\mathrm{k}}$ constitutes the associate point of $\mathrm{P}_{\mathrm{k}-1}$. More details about how to find the association between consecutive images is available in [14][13].

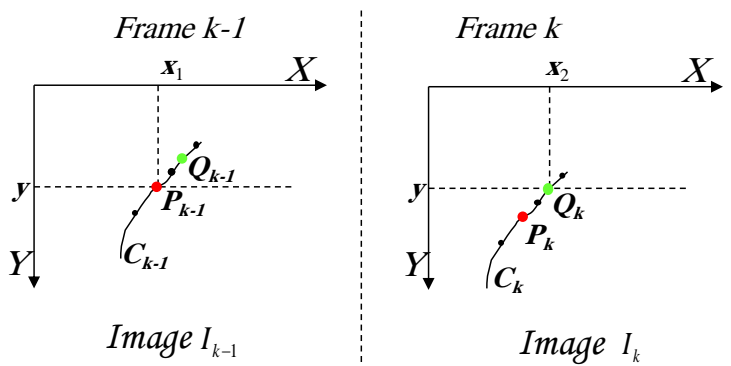

Fig 1: $I_{k-1}$ and $I_{k}$ represent consecutive images. The point $Q_{k}$ in the image $I_{k}$ constitutes the associate point of the point $P_{k-1}$ in the image $I_{k-1}$.

\subsubsection{Temporal correspondence}

In this subsection, we illustrate how to find the correspondence between edge curves of consecutive images based on the association computed in the previous subsection. Temporal correspondence consists then of finding for each edge curve in the current image its corresponding one in the previous, if it exists. A connected edge points in the current image must associate to the connected ones in the previous image, which it shares the greatest associated points, this constraint is satisfied by using the method of Winner-takes-all (WTA) on each curve (Fig 2), then many false associated points are corrected. This can be explained by the associated points should belong to the same object contour and they should have similar or closer gradient magnitudes and orientation. In this work, we use an important cost function (Eq 1.) described below in this paper. This function computes the distance between two candidate associate points using gradient magnitudes. The edge with smaller cost will be considered as associated pairs of features. The association approach does not guarantee that each feature in the image have its associated point. But some good associate points are enough to match two curves.
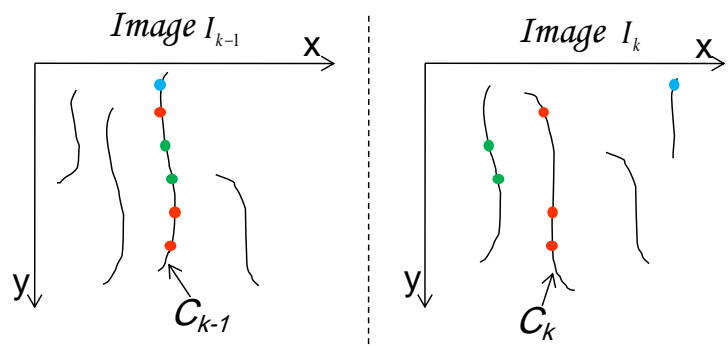

Time

Fig 2: Edge curves correspondence using association between edge points of consecutive images, associate edge points can be belonging to different curves. The WTA algorithm will be used to correct false associated edge points.

\subsubsection{Cost function}

As a similarity criterion between corresponding edge points, we use a cost function based on the gradient magnitude and orientation at the matched edge points. Let $\mathrm{e}_{\mathrm{k}-1}$ and $\mathrm{e}_{\mathrm{k}}$ be two edge points belonging to images $\mathrm{I}_{\mathrm{k}-1}$ and $\mathrm{I}_{\mathrm{k}}$, respectively. We denote by $\mathrm{m}_{\mathrm{k}-1}$ and $\mathrm{m}_{\mathrm{k}}$ (resp. $\mathrm{O}_{\mathrm{k}-1}$ and $\mathrm{O}_{\mathrm{k}}$ ) their gradient magnitudes (resp. orientations), respectively. We assume that associate edge points on the consecutive images should have the same (or closer) gradient magnitudes as well as the same (or close) orientations. Therefore, we define the cost function as follows. 


$$
\begin{array}{r}
C\left(e_{k-1}, e_{k}\right)=\left\{\left(I_{k-1}(x, y)-I_{k}\left(x^{\prime}, y\right)\right)^{2}+\left(m_{k-1}\right)^{2}+\right. \\
\left.\left(m_{k}\right)^{2}-2 * m_{k-1} * m_{k} * \cos \left(O_{k-1}-O_{k}\right)\right\}^{1 / 2}
\end{array}
$$

Where $(\mathrm{x}, \mathrm{y})$ and $\left(\mathrm{x}^{\prime}, \mathrm{y}\right)$ are the coordinates of the edge points $\mathrm{e}_{\mathrm{k}-1}$ and $\mathrm{e}_{\mathrm{k}}$, respectively.

\section{PEDESTRIAN DETECTION}

Here we describe the proposed method for pedestrian detection. The proposed approach is subdivided into many steps as shown in Fig.3. in this work we used a fast motion detector algorithm for obtaining horizontal motion velocity for each edge curves. Then a horizontal-vertical projection histogram has been used for detecting the ROIs (Region of Interest). Finally a Adaboost classifier has been applied for each $R O I$ to validate detected object (Pedestrian or not).

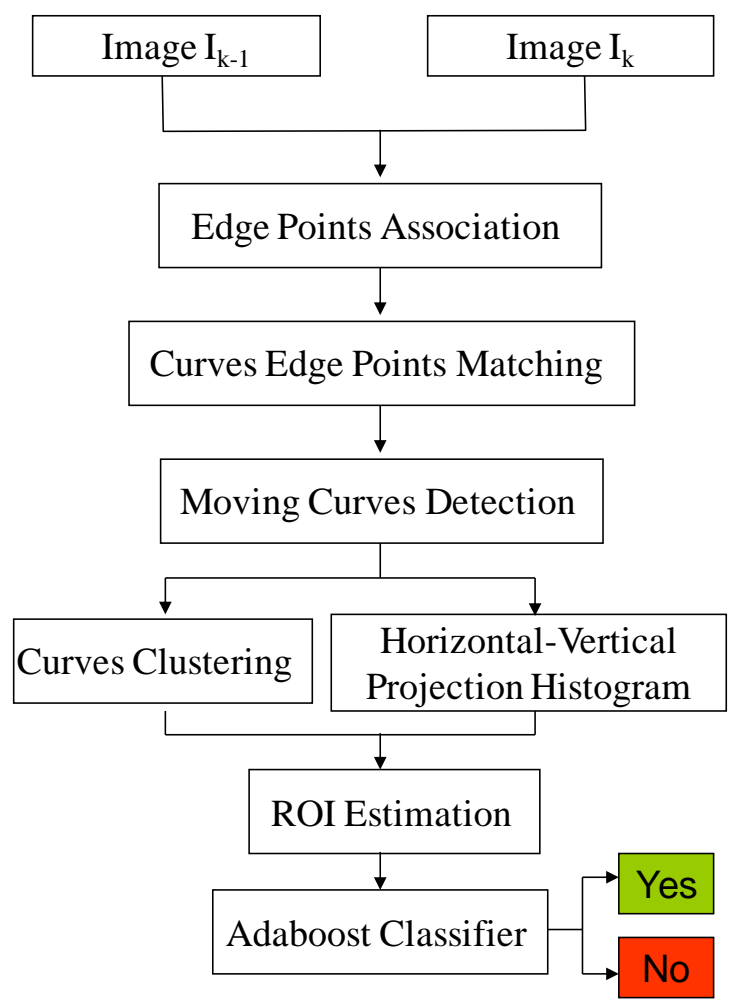

Fig 3: Pedestrian detection algorithm.

\subsection{Moving objects detection}

We propose a motion-based attention mechanism to improve the performance of pedestrian recognition system, this attentive strategy utilizes horizontal motionof curves, for each curve in current image we compute displacement velocity with the previous image. Therefore, we used the representative motion vectors found for each curve to detect moving objects, and then the ROIs (Region Of Interest) should be found for each moving object, for reach to this purpose the horizontal and vertical projection histogram has been used. Fig.4(f) and Fig.5(left) show the projection histogram.

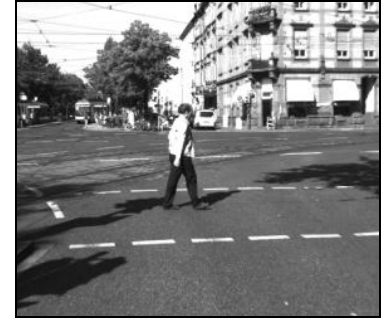

(a)

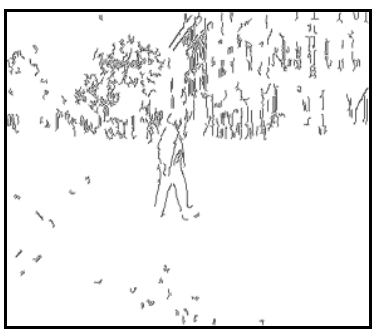

(c)

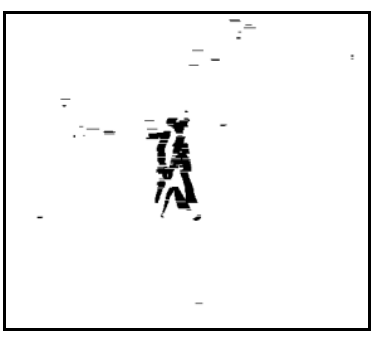

(e)

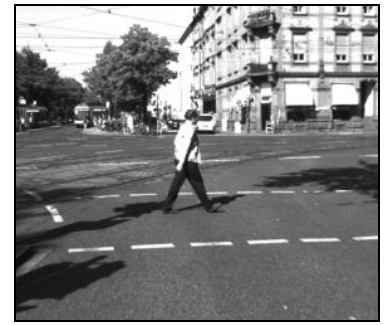

(b)

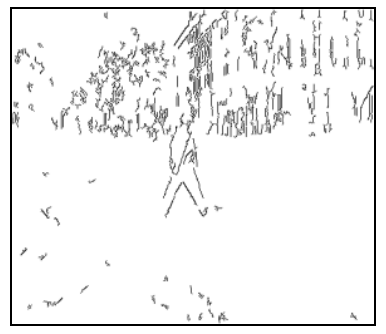

(d)

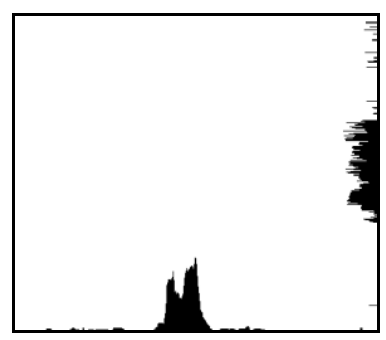

(f)
Fig 4: Results of moving object detection. (a) input image at time k-1, (b) input image at time $k$, (c) and (d) veridical edge curves of image (a) and (b) respectively.

(e) horizontal motion vectors using association approach, (f) Horizontal-vertical histogram projection.

\subsection{ROIs Estimation}

Until this step the moving objects have been detected. Now we should localize ROIs. To achieve this task we propose to use the Horizontal-vertical histogram projection obtained in previous section. We concentrate only where significant motion is detected. This significant motion is presented by closest picks in histogram. With horizontal-vertical projection the ROIs can be easily obtained. Fig.5 shows $R O I$ extraction.
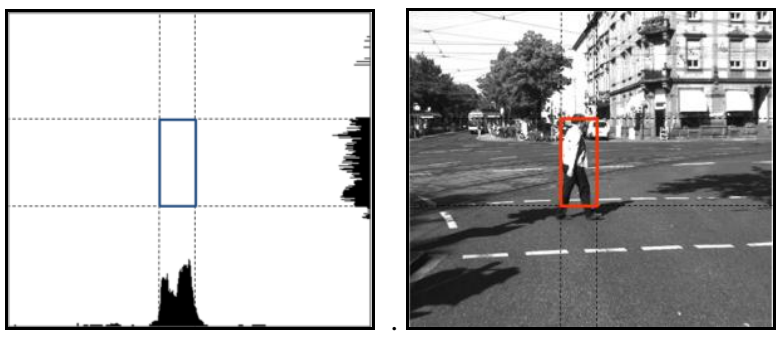

Fig 5: ROI localization. (left) intersection of vertical and horizontal significant motion. (right) ROI at the reel image.

\subsection{Validation and Classifier}

The literature propose many techniques to classify and validate detecting object, in this work we have tested two 
methods, the first one use the ratio of height to width for each detecting $R O I$, this ratio has been used for omitting some object which do not respect pedestrian form. The second method which is the last step in our pedestrian detection system use Adaboost Classifier to validate detecting object. In order to make our system detection very fast as possible, ROIs detected have been used as sub-search area for Adaboost Classifier process.

\section{EXPERIMENTAL RESULT}

This section presents the results obtained when we applied the proposed algorithm to real image sequences. The images have a size of 450x391 (obtained from Karlsruhe Dataset [3][1]). In order to evaluate the performance of our approach, we have experimented with many other image sequences (Fig.6) acquired by camera mounted in a moving road vehicle. The detection rate is high, and our approach proves to be reliable and be able to detect most pedestrian in city traffic. Our method is very performed for pedestrians crossing the road. The hardware used for the experiments is a HP Intel(R) Core(TM)2 Duo CPU 2.09GHZ running under Windows XP.

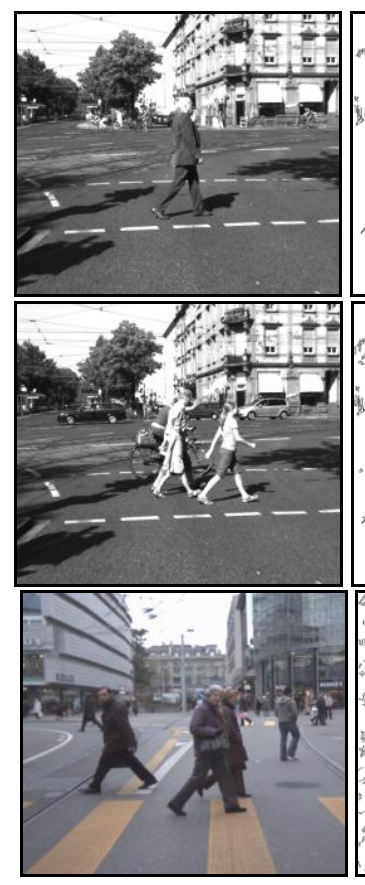

(a)

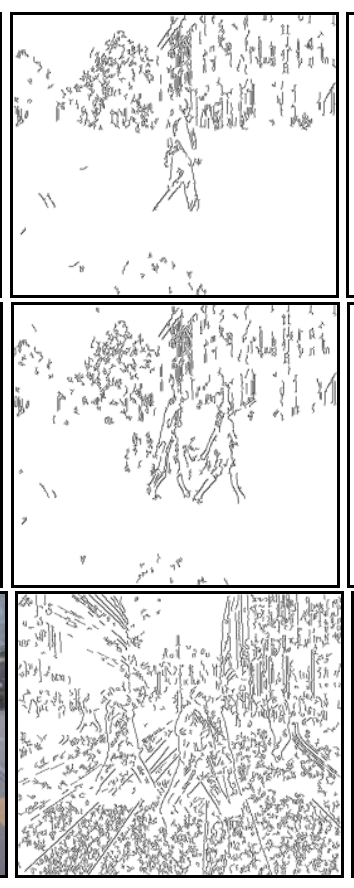

(b)

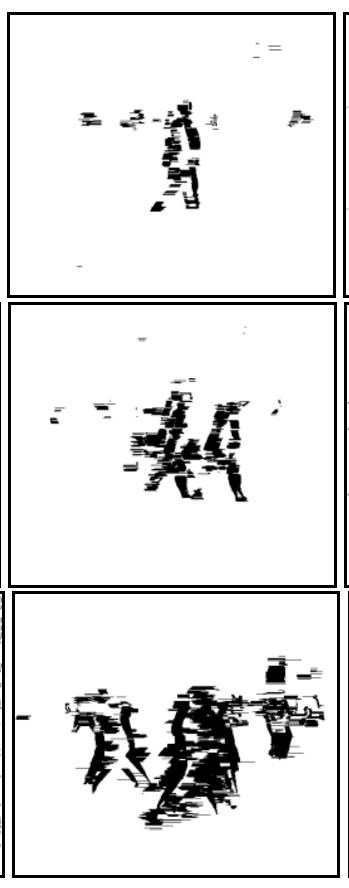

(c)

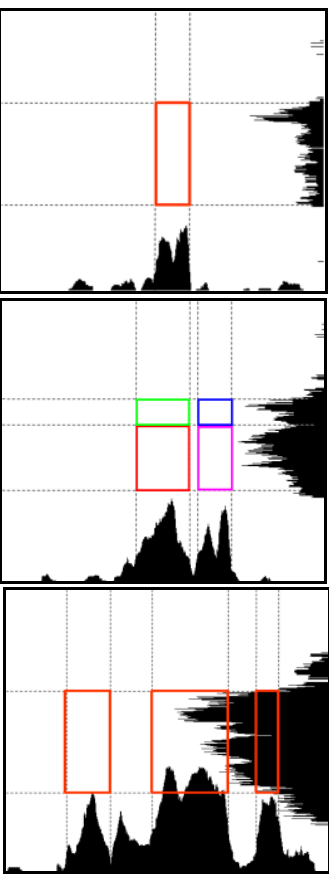

(d)

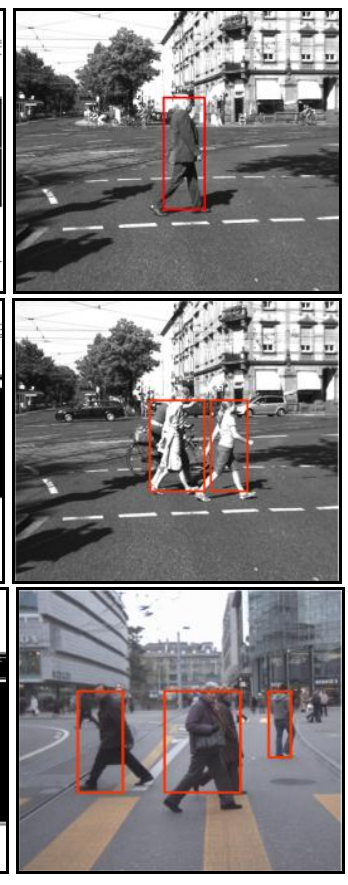

(e)

Fig 6: Pedestrian detection results using our approach, (a) shows the input image, (b) vertical edge curves using Canny operator, (c) vertical vector motion using association approach [14][13], (d) horizontal-vertical histogram motion projection and ROIs detection, (e) detection of moving objects (pedestrians).

\section{CONCLUSION}

Our approach has been proposed to detect pedestrian crossing street in front of vehicle. The method is dedicated to intelligent vehicle applications. The proposed method is very fast, due to the association method between edge curves of successive images. The time can be reduced more by using GPU card for our implementations. This new method has been tested on different real image sequence and the results are satisfactory. We started by finding association between edge points and then corresponding curves of successive frames. Good cost function is used for this task. Then localization of ROIs is achieved by using horizontal and vertical projection histogram. Finally, the Adaboost classifier is applied on the RIOs provided by last step. In the future work, we plan to improve this step of proposed method. The eventual future technique should provide more accurate pedestrian detection and need less running time.

\section{REFERENCES}

[1] Andreas Geiger and Martin Roser and Raquel Urtasun, "Efficient Large-Scale Stereo Matching", Asian Conference on Computer Vision,2010 November, Queenstown, New Zealand.

[2] B. Wu and R. Nevatia. "Cluster boosted tree classifier for multi-view, multi-pose object detection". In ICCV, 2007.

[3] Bernd Kitt and Andreas Geiger and Henning Lategahn, "Visual Odometry based on Stereo Image Sequences with RANSAC-based Outlier Rejection Scheme", IEEE Intelligent Vehicles Symposium, 2010 June, San Diego, USA.

[4] Broggi, A. F, "stereo-based preprocessing for human shape localization in unstructured environments" In Proc. of the IEEE Intelligent Vehicle Symposium., pp. 410415, Ohio USA, 2003. 
[5] C. J. C. Burges. "A tutorial on support vector machines for pattern recognition". Data Mining and Knowledge Discovery, 2:121-167, 1998.

[6] D. M. Gavrila and S. Munder. "Multi-cue pedestrian detection andtracking from a moving vehicle". IJCV, pages 41-59, 2007.

[7] E. Seemann, M. Fritz, and B. Schiele. "Towards robust pedestrian detection in crowded image sequences". In CVPR, 2007.

[8] Gavrila, D., "Sensor-based pedestrian protection", IEEE Intelligent Systems, pp. 77-81, 2001.

[9] J. Canny, "A computational approach to edge detection", IEEE Trans.Pattern Anal. Mach. Intell., vol. PAMI-8, no. 6, pp. 679-698, Nov. 1986.

[10] Kurita, K. N. "Boosting Soft-Margin SVM with Feature Selection for Pedestrian Detection", Springer-Verlag Berlin Heidelberg , pp. 22-31, 2005.

[11] M. Bertozzi, "Infrared Stereo Vision-based Pedestrian DetecIntelligent Vehicles Symposium", 2005, pp. 23-28. Parma:IEEE.

[12] M. El Ansari, Abdenbi Mazoul, Abdelaziz Bensrhair, George Bebis, "A Real-time Spatio-Temporal Stereo Matching for Road Applications", 2011 14th International IEEE Conference on Intelligent Transportation Systems Washington, DC, USA. October 5-7, 2011.

[13] M. El-Ansari, S. Mousset, A. Bensrhair, and G. Bebis, "Temporal consistent fast stereo matching for adavnced driver assistance systems (ADAS) ", in Proc. of IEEE Intelligent Vehciles Symposium, San Diego, CA, USA, June 21-24 2010, pp. 825-831.

[14] M. El-Ansari, S. Mousset, and A. Bensrhair, "Temporal consistent real-time stereo for intelligent vehicles", Pattern Recognition Letters, vol. 31, no. 11, pp. 12261238, August 2010.
[15] Munder, D. M, "An Experimental Study on Pedestrian Classification", IEEE Transactions on Pattern Analysis and Machine Intelligence. 2006,pp. 1863-1868.

[16] P. Sabzmeydani and G. Mori. "Detecting pedestrians by learning shapelet features". In CVPR, 2007.

[17] P. Viola and M. Jones, "Rapid object detection using a boosted cascade of simple features", in CVPR, 2001.

[18] P. Viola and M. Jones. "Robust real-time object detection". IJCV, 57(2):137-154, 2004.

[19] P. Viola, M. J., "Stereo- and Neural Network-Based Pedestrian Detection", IEEE Trans. Intelligent Transportation Systems, vol 1, no. 3, 2000.

[20] P. Viola, M. Jones, and D. Snow. "Detecting pedestrians using patterns of motion and appearance". In CVPR, 2003.

[21] R. E. Schapire, "The boosting approach to machine learning: An overview", In MSRI Workshop on Nonlinear Estimation and Classification, 2002, 2002.

[22] S. Munder, C. Schn"orr, and D. Gavrila. "Pedestrian detection and tracking using a mixture of view-based shape-texture models". In IEEE Transactions on Intelligent Transportation Systems, 2008.

[23] Thorpe, L. ,"Stereo- and Neural Network-Based Pedestrian Detection", IEEE Trans. Intelligent Transportation Systems, vol 1, no. 3,2000.

[24] Zebbara Khalid , Abdenbi Mazoul, Mohamed El Ansari "A new vehicle detection method", International Journal of Advanced Computer Science and Applications (IJACSA), Special Issue on Artificial Intelligence, Volume 2 No. 8 August 2011.

[25] Zebbara Khalid, Abdenbi Mazoul, Mohamed El Ansari, "On Road Vehicle Detection using Association Approach", International Journal of Computer Applications (IJCA) (0975 - 8887) Volume 34- No.2, November 2011. 